\title{
Adult Onset Vitiligo: Multivariate Analysis Suggests the Need for a Thyroid Screening
}

\author{
L. Lazzeri, R. Colucci, A. Cammi, F. Dragoni, and S. Moretti \\ Department of Surgery and Translational Medicine, Section of Dermatology, University of Florence, Florence, Italy \\ Correspondence should be addressed to R. Colucci; roberta.colucci21@gmail.com
}

Received 25 July 2016; Accepted 30 August 2016

Academic Editor: Davinder Parsad

Copyright ( 2016 L. Lazzeri et al. This is an open access article distributed under the Creative Commons Attribution License, which permits unrestricted use, distribution, and reproduction in any medium, provided the original work is properly cited.

\begin{abstract}
Background. There are limited epidemiological studies evaluating the effect of age at onset on disease features in vitiligo. Objectives. To identify factors associated with adult onset vitiligo in comparison with childhood onset vitiligo. Patients and Methods. We retrospectively collected medical records of 191 patients. Such records included clinical examination, personal and familial medical history, laboratory evaluations, concomitant vitiligo treatment and drug assumption. Results. 123 patients with a disease onset after the age of 40 (adult onset vitiligo) were compared with 68 patients who developed vitiligo before the age of 12 (childhood onset vitiligo). Multivariate analysis revealed that personal history of thyroid diseases $(P=0.04$; OR 0.4$)$, stress at onset $(P=0.002$; OR $=0.34)$, personal history of autoimmune thyroid disease (ATD) $(P=0.003$; OR $=0.23)$, and thyroid nodules $(P=0.001$; OR 0.90$)$ were independently associated with adult onset vitiligo, whereas family history of dermatological diseases $(P=0.003$; OR $=2.87)$ and Koebner phenomenon $(P<0.001 ; \mathrm{OR}=4.73)$ with childhood onset vitiligo. Moreover, in the adult onset group, concomitant thyroid disease preceded vitiligo in a statistically significant number of patients $(P=0.014)$. Conclusions. Childhood onset and adult onset vitiligo have different clinical features. In particular, ATD and thyroid nodules were significantly associated with adult onset vitiligo, suggesting that a thyroid screening should be recommended in this group of patients.
\end{abstract}

\section{Introduction}

Vitiligo is a chronic acquired pigmentary skin disorder, clinically characterized by well-defined asymptomatic white macules, which are the result of a loss of functional melanocytes in the epidermis. It is considered the most common pigmentary disorder, affecting $0.1-2 \%$ of the world population, with no sexual or racial preference [1].

Vitiligo can appear at any age, but it has been most frequently observed in the first two decades [2].

In particular, it tends to appear before the age of 10 in about $25 \%$ of the patients [3,4]. However, a disease onset during adulthood is a common condition as well [5], even if to date only few studies $[6,7]$ have described the clinic profile of adult onset vitiligo.

We herein present the results of a retrospective observational study comparing the clinical characteristics of adult and childhood onset vitiligo through univariate analysis and multivariate conditional logistic regression.

\section{Patients and Methods}

We retrospectively collected medical records of 500 consecutive patients diagnosed with vitiligo attending the Florence University specialized vitiligo outpatient service between June 2010 and June 2013. Patients with vitiligo arisen between the ages of 12 and 40 years were excluded from the study. The remaining 191 patients were divided into two groups, according to the age at onset of the disease: the adult onset group including 123 patients with a disease onset after the age of 40 and the childhood onset group including 68 patients, both children and adults, having a disease onset before the age of 12 years. 
The study design was conducted according to the Declaration of Helsinki principles and informed consent was obtained from each patient.

2.1. Medical Assessment. Medical records included clinical examination, personal and familial medical history, laboratory evaluations, and concomitant vitiligo treatment and drug assumption.

Medical assessment was performed using a modified VETF form [8, 9]. This evaluation individually assesses four body regions (head and neck, trunk, upper extremities, and lower extremities), examined both under natural light and under Wood lamp, for the extent of depigmentation (affected body surface percentage scored 0-100\%), stage of disease (staging), scored 0-4 (normal pigmentation, incomplete depigmentation, complete depigmentation, complete depigmentation plus partial leukotrichia, and complete depigmentation plus complete leukotrichia), and disease progression (spreading), scored -1 to +1 (ongoing subclinical repigmentation, similar limits, and ongoing subclinical depigmentation). A total score for the aforementioned parameters was calculated with values ranging from 0 to 16 for staging and -4 to +4 for spreading.

The following demographic and clinical information was taken into consideration: sex, age, age at onset of the disease, phototype, Koebner phenomenon, presence of Sutton naevi, personal history of premature hair graying (more than $50 \%$ of white hair before the age of 40 years), emotional stress as a triggering factor, previous episodes of repigmentation, inflammation/pruritus of the macules, and modality of onset of vitiligo.

We also evaluated personal and familial history of autoimmune diseases (pernicious anemia, Addison's disease, systemic lupus erythematosus, inflammatory bowel disease, alopecia areata, multiple sclerosis, myasthenia gravis, rheumatoid arthritis, scleroderma, Sjogren's syndrome, coeliac disease), thyroid disorders (in general and specifically autoimmune thyroid disorders (ATD) or nodules), cardiovascular, psychiatric, and neurological diseases, diabetes, hypertension, and dermatological diseases (in general and specifically psoriasis, atopic dermatitis, and melanoma). Familial history of vitiligo was also investigated.

Laboratory examinations included serum levels of triiodothyronine (T3) and thyroxine (T4) thyroid stimulating hormone (TSH) and the following autoantibody titers: antithyroglobulin (anti-TG), anti-thyroperoxidase (anti-TPO), anti-TSH receptor (anti-TSHR), anti-parietal cell antibody (APCA), anti-smooth muscle antibody (ASMA), anti-nuclear antibody (ANA), and extractable nuclear antibody (ENA).

2.2. Statistical Analysis. Characteristics of the study population were described using mean, standard deviations, frequencies, and percentages. In order to recognize factors associated with an age of onset $>40$ years and an age of onset $<12$ years, comparisons between groups were conducted by univariate and multivariate conditional logistic regression. Every possible potential predictor of the above-mentioned age of onset was firstly assessed individually, using the chi
square/Fisher tests for categorical data and Student's $t$-test for quantitative data in a univariate analysis. The odds ratios (OR), the corresponding 95\% confidence intervals (CIs), and $P$ values were computed. Secondarily, every predictor was assessed using multivariate analysis, with odds ratios and associated $95 \%$ confidence intervals.

All $P$ values $<0.05$ were considered to be statistically significant. Statistical analyses were performed using the SPSS 15.0 for Windows program (Statistical Package for Social Science, SPSS Inc., Chicago, IL, USA).

\section{Results}

Patient demographics and clinical characteristics are shown in Table 1.

Results of the univariate analysis for patient characteristics in the adult onset group versus childhood onset disease revealed that phototype III (OR 0.49; $P=0.021$ ), personal history of hypertension (OR 0.8; $P<0.001$ ), head and neck involvement at disease onset $(P=0.007)$, and stress as an onset factor (OR $0.29 ; P<0.001$ ) were all positively associated with adult onset vitiligo. Moreover this group showed a significantly higher prevalence of thyroid disease (OR 0.27 ; $P<0.001$ ), ATD (OR 0.22; $P=0.02$ ), and thyroid nodules (OR 0.9; $P=0.002$ ).

On the contrary, family history of dermatological conditions (OR 2.615; $P=0.002$ ), family history of autoimmune diseases (OR 3.96; $P=0.020$ ), phototype II (OR 2.02; $P=0.021)$, initial localization of the disease involving upper extremities $(P=0.009)$, and the presence of Koebner phenomenon (OR 3.00; $P<0.001$ ) were all positively associated with childhood onset. Complete results of the univariate statistical analysis are listed in Table 1.

The results of the multivariate analysis pointed out that a family history of dermatological diseases $(P=0.003, \mathrm{OR}$ $2.87)$ and Koebner phenomenon $(P<0.001$, OR 4.73) were independently associated with an age of onset of vitiligo $<12$ years (Table 2). In contrast, stress at onset $(P=0.002, \mathrm{OR}$ $0.34)$, personal history of thyroid diseases $(P=0.04$, OR 0.4$)$, ATD $(P=0.003$, OR 0.23$)$, and thyroid nodules $(P=0.001$, OR 0.90 ) were independently associated with vitiligo age of onset $>40$ years.

In light of this data, we performed a further analysis in our patients affected by both vitiligo and thyroid disease, in order to evaluate whether or not the onset of vitiligo appeared prior to thyroid disease. According to our results, in the adult onset group, thyroid disease preceded vitiligo in a statistically significant number of patients $(P=0.014)$. However no statistical association was found when we performed this analysis considering ATD and thyroid nodules separately.

\section{Discussion}

Until now, there is a lack of information about the clinical and epidemiologic profile of adult onset vitiligo and only few studies have compared its characteristics with early onset disease [10-12]. The purpose of our study was to describe the effect of age at onset on disease features. 
TABLE 1: Patients characteristics and complete results of the univariate analysis.

\begin{tabular}{|c|c|c|c|c|}
\hline Parameter $(*)$ & Age of onset $<12$ years & Age of onset $>40$ years & $P$ & OR \\
\hline Mean age at onset & 7 years (range $0-12$ ) & 50.25 years (range $40-83$ ) & & \\
\hline Mean age at time of consultation & 19.5 years (range $3-69$ ) & 56.3 years (range $41-85$ ) & & \\
\hline Mean duration of the disease & 12.2 years (range $0.3-62$ ) & 6.2 years (range $0.2-30$ ) & & \\
\hline \multicolumn{5}{|l|}{ Sex } \\
\hline Female & $34(50.0 \%)$ & $68(55.2 \%)$ & 0.438 & \multirow{2}{*}{1.236} \\
\hline Male & $34(50.0 \%)$ & $55(44.8 \%)$ & & \\
\hline \multicolumn{5}{|l|}{ Type } \\
\hline Segmental & $4(5.9 \%)$ & $4(3.3 \%)$ & 0.463 & 0.552 \\
\hline Nonsegmental & $62(91.2 \%)$ & $116(96.6 \%)$ & 0.173 & 2.806 \\
\hline Unclassified & $2(2.9 \%)$ & 0 & 0.130 & 0.355 \\
\hline \multicolumn{5}{|l|}{ Family history } \\
\hline Vitiligo & $22(32.3 \%)$ & $25(24.3 \%)$ & 0.247 & 0.670 \\
\hline Psoriasis & $17(25.0 \%)$ & $15(14.5 \%)$ & 0.087 & 0.511 \\
\hline Melanoma & $4(5.8 \%)$ & $6(5.8 \%)$ & 0.988 & 0.990 \\
\hline Dermatological & $36(52.9 \%)$ & $37(34.2 \%)$ & 0.002 & 2.615 \\
\hline Thyroid disease & $23(33.8 \%)$ & $29(26.8 \%)$ & 0.324 & 0.718 \\
\hline Cardiovascular disease & $9(13.2 \%)$ & $10(10.0 \%)$ & 0.516 & 0.728 \\
\hline Type I diabetes & $1(1.4 \%)$ & $5(4.9 \%)$ & 0.231 & 3.490 \\
\hline Type II diabetes & $12(17.6 \%)$ & $16(15.8 \%)$ & 0.921 & 0.878 \\
\hline Autoimmune disease & $8(11.7 \%)$ & $4(4.0 \%)$ & 0.020 & 3.96 \\
\hline Hypertension & $14(20.5 \%)$ & $13(12.8 \%)$ & 0.179 & 0.570 \\
\hline Neurologic disease & $0(0.0 \%)$ & $1(1.0 \%)$ & 0.406 & 0.590 \\
\hline \multicolumn{5}{|l|}{ Personal history of } \\
\hline Thyroid disease & $13(19.1 \%)$ & $40(37.7 \%)$ & $<0.001$ & 0.27 \\
\hline ATD & $7(10.6 \%)$ & $27(25.0 \%)$ & 0.020 & 0.22 \\
\hline Thyroid nodules & $0 \%$ & $13(12.0 \%)$ & 0.002 & 0.90 \\
\hline Cardiovascular disease & $3(4.4 \%)$ & $5(5.0 \%)$ & 1.000 & 1.152 \\
\hline Autoimmune disease & $3(4.4 \%)$ & $7(7.0 \%)$ & 0.742 & 1.649 \\
\hline Type I diabetes & $0(0.0 \%)$ & $2(2.0 \%)$ & 0.515 & 0.590 \\
\hline Type II diabetes & $0(0.0 \%)$ & $3(3.0 \%)$ & 0.273 & 0.588 \\
\hline Hypertension & $0(0.0 \%)$ & $18(18.1 \%)$ & $<0.001$ & 0.8 \\
\hline Psychiatric disease & $0(0.0 \%)$ & $3(3.0 \%)$ & 0.273 & 0.588 \\
\hline Neurologic disease & $1(1.5 \%)$ & $2(2.0 \%)$ & 1.000 & 1.367 \\
\hline \multicolumn{5}{|l|}{ Phototype } \\
\hline $\mathrm{I}$ & $1(1.5 \%)$ & $0(0.0 \%)$ & 0.364 & 0.360 \\
\hline II & $36(52.9 \%)$ & $44(36.9 \%)$ & 0.034 & 0.521 \\
\hline III & $28(41.2 \%)$ & $68(62.4 \%)$ & 0.036 & 1.905 \\
\hline IV & $2(2.9 \%)$ & $7(5.9 \%)$ & 0.491 & 2.063 \\
\hline $\mathrm{V}$ & $1(1.5 \%)$ & $0(0.0 \%)$ & 0.364 & 0.360 \\
\hline \multicolumn{5}{|l|}{ Previous episode of repigmentation } \\
\hline Spontaneous & $4(5.9 \%)$ & $8(8.0 \%)$ & 0.764 & 1.376 \\
\hline Drug induced & $27(39.7 \%)$ & $33(32.9 \%)$ & 0.349 & 0.737 \\
\hline Photoexposition & $9(13.2 \%)$ & $9(9.0 \%)$ & 0.372 & 0.641 \\
\hline \multicolumn{5}{|l|}{ Type of Onset } \\
\hline Sudden & $30(44.1 \%)$ & $57(48.7 \%)$ & 0.618 & 1.165 \\
\hline Progressive & $35(51.5 \%)$ & $62(52.1 \%)$ & 0.934 & 1.026 \\
\hline Leucotrichia & $16(23.5 \%)$ & $35(36.0 \%)$ & 0.086 & 1.835 \\
\hline Sutton & $8(11.7 \%)$ & $7(8.9 \%)$ & 0.580 & 0.739 \\
\hline
\end{tabular}


TABLE 1: Continued.

\begin{tabular}{lccrc}
\hline Parameter $(*)$ & Age of onset $<12$ years & Age of onset $>40$ years & OR \\
\hline Koebner & $42(61.7 \%)$ & $43(43.8 \%)$ & $<$ & $<\mathbf{0 . 0 0 1}$ \\
\hline Stress as onset factor & $29(42.6 \%)$ & $67(65.0 \%)$ & $<\mathbf{0 . 0 0 1}$ & $\mathbf{2 . 5 0 3}$ \\
\hline
\end{tabular}

${ }^{*}$ Continuous variables such as age, age of onset, and vitiligo duration are reported as mean \pm SD and expressed in years. For every other parameter evaluated, we report the number and the percentage of patients positive or negative for that parameter.

TABLE 2: Significant results of the multivariate conditional logistic regression.

\begin{tabular}{lcc}
\hline Parameter & $P$ & OR \\
\hline Family history of dermatological disease & 0.003 & 2.87 \\
Personal thyroid diseases & 0.04 & 0.4 \\
Koebner & $<0.001$ & 4.73 \\
Stress as onset factor & 0.002 & 0.34 \\
Personal ATD & 0.003 & 0.23 \\
Personal thyroid nodules & 0.001 & 0.90 \\
\hline
\end{tabular}

In our study, both sexes were equally affected and we did not find any significant difference in sex ratio $(\mathrm{M}: \mathrm{F}, 1: 1)$ between adult and childhood onset vitiligo. This result is in accordance with two previous papers $[3,13]$. However, a female preponderance has been reported in childhood onset, compared with adult onset, vitiligo $[4,14]$. Other studies $[11,12]$ comparing disease characteristics in childhood and later onset vitiligo (disease onset after the age of 12) reported a female preponderance in both groups.

The role of stress as an onset factor in vitiligo has been widely investigated, both in adults and in children $[15,16]$. In our study patients with adult onset vitiligo recalled a stressful event with significantly higher frequency compared to those with childhood onset. This result is in agreement with the above-mentioned studies, regarding childhood and later onset vitiligo [11, 12]. However, according to Ezzedine et al. [11] patients with childhood onset vitiligo might be less able to recall such an event when seen first in adulthood, so a recall bias could be responsible for this difference.

Among the other significant results, we found a significantly higher prevalence of thyroid disease, ATD, and thyroid nodules in adult onset vitiligo patients. With regard to ATD, the reported diagnosis was confirmed by positive serum antiTPO, anti-TG, anti-TSHR, and suggestive ultrasonography. The association between ATD and vitiligo has been widely explored in children [17]. On the contrary little is known about this relationship in adult onset vitiligo. As reported in a recent systemic review [18], patients with vitiligo show a 2.5fold higher risk of developing ATD compared with patients without vitiligo. The risk of developing ATD seems to increase with age and this is in accordance with the assumption of the increase in prevalence of autoimmunity with age [19]. This could explain the statistical association assessed by our study, since mean age in adult onset group is higher than in the childhood onset one. Our results are also in agreement with the paper of Nicolaidou et al. [12], claiming that the risk of developing ATD was significantly lower when vitiligo appeared during childhood.
We also found an association between adult onset disease and thyroid nodules which, to our knowledge, has never been studied so far. This relationship could be due to the higher age of this group (56.3 yr versus $19.5 \mathrm{yr}$ ), which is considered an important risk factor for thyroid nodules [20].

Analyzing the age at onset of vitiligo compared with thyroid disease in patients affected by both diseases we found that, in the adult onset group, thyroid disease tends to precede vitiligo in a significant number of patients. According to recent studies [21, 22] ATD is associated with an increased systemic oxidative stress, which has been shown to play a major role in the pathogenesis of vitiligo [23]. In our analysis the relationship between adult onset vitiligo and previous thyroid disease was confirmed only when thyroid disease was considered on the whole, since statistical significance was lost when ATD and nodules were considered separately. However it should be considered that $68 \%$ of the cases of thyroid diseases we considered in our analysis were actually ATD, so it is possible that the loss of significance considering ATD alone could be related to the smaller number of patients.

Our analysis also revealed a significantly higher prevalence of Koebner phenomenon in the childhood onset group $(P<0.001)$, which had not been observed in other studies. A possible explanation for these figures could be that children are more likely to undergo skin traumas while playing and therefore to develop koebnerization of the skin. However our childhood onset group also included adults so other factors might be involved in this phenomenon.

Patients with childhood onset vitiligo were also more likely to have a positive history of dermatological diseases in their family, but when dermatological diseases were analyzed individually, no significant association between childhood vitiligo and a family history of vitiligo, melanoma, or psoriasis was found. Personal and familial history of atopic dermatitis were not associated with childhood onset vitiligo, in contrast with Ezzedine et al. [11]. This difference could be due to a recall error, since atopic dermatitis usually develops in the first year of life, and the majority of affected individuals have resolution of disease by adulthood [24].

The limitations of the study include possible recall bias, especially in patients whose disease started many years before presentation to our clinic.

\section{Conclusions}

In conclusion, upon multivariate conditional regression, our study shows that adult and childhood onset vitiligo have different clinical features. Childhood onset vitiligo is significantly associated with family history of dermatological conditions and Koebner phenomenon, whereas adult onset 
vitiligo is associated with stress as an onset factor, thyroid disease, ATD, and thyroid nodules. Those differences must be kept in due consideration in order to suggest appropriate screening programs and follow-up; thyroid screening in particular should be suggested to all patients with vitiligo, but its importance is even stronger in adult onset vitiligo, since the risk of developing thyroid disease is higher in these patients.

\section{Competing Interests}

The authors have no financial obligations or conflict of interests to declare.

\section{References}

[1] C. Krüger and K. U. Schallreuter, "A review of the worldwide prevalence of vitiligo in children/adolescents and adults," International Journal of Dermatology, vol. 51, no. 10, pp. 1206-1212, 2012.

[2] S. Handa and I. Kaur, "Vitiligo: clinical findings in 1436 patients," Journal of Dermatology, vol. 26, no. 10, pp. 653-657, 1999.

[3] Z. Hu, J.-B. Liu, S.-S. Ma, S. Yang, and X.-J. Zhang, "Profile of childhood vitiligo in China: an analysis of 541 patients," Pediatric Dermatology, vol. 23, no. 2, pp. 114-116, 2006.

[4] S. Handa and S. Dogra, "Epidemiology of childhood vitiligo: a study of 625 patients from North India," Pediatric Dermatology, vol. 20, no. 3, pp. 207-210, 2003.

[5] J. Howitz, H. Brodthagen, M. Schwartz, and K. Thomsen, "Prevalence of vitiligo. Epidemiological survey on the Isle of Bornholm, Denmark," Archives of Dermatology, vol. 113, no. 1, pp. 47-52, 1977.

[6] S. Dogra, D. Parsad, S. Handa, and A. J. Kanwar, "Late onset vitiligo: a study of 182 patients," International Journal of Dermatology, vol. 44, no. 3, pp. 193-196, 2005.

[7] A. J. Kanwar, R. Mahajan, and D. Parsad, "Effect of age at onset on disease characteristics in vitiligo," Journal of Cutaneous Medicine and Surgery, vol. 17, no. 4, pp. 253-258, 2013.

[8] S. Moretti, M. Arunachalam, R. Colucci et al., "Autoimmune markers in vitiligo patients appear correlated with obsession and phobia," Journal of the European Academy of Dermatology and Venereology, vol. 26, no. 7, pp. 861-867, 2012.

[9] M. Arunachalam, R. Colucci, S. Berti et al., "Autoimmune signals in non-segmental vitiligo patients are associated with distinct clinical parameters and toxic exposures," Journal of the European Academy of Dermatology and Venereology, vol. 27, no. 8, pp. 961-966, 2013.

[10] H. E. Teulings, E. Ceylan, M. Overkamp et al., "Nonsegmental vitiligo disease duration and female sex are associated with comorbidity and disease extent: a retrospective analysis in 1307 patients aged $\geq 50$ years," British Journal of Dermatology, 2016.

[11] K. Ezzedine, A. Diallo, C. Léauté-Labrèze et al., "Pre- vs. postpubertal onset of vitiligo: multivariate analysis indicates atopic diathesis association in pre-pubertal onset vitiligo," British Journal of Dermatology, vol. 167, no. 3, pp. 490-495, 2012.

[12] E. Nicolaidou, C. Antoniou, A. Miniati et al., "Childhood- and later-onset vitiligo have diverse epidemiologic and clinical characteristics," Journal of the American Academy of Dermatology, vol. 66, no. 6, pp. 954-958, 2012.
[13] S. Cho, H.-C. Kang, and J.-H. Hahm, "Characteristics of vitiligo in Korean children," Pediatric Dermatology, vol. 17, no. 3, pp. 189-193, 2000.

[14] U. Pajvani, N. Ahmad, A. Wiley et al., "The relationship between family medical history and childhood vitiligo," Journal of the American Academy of Dermatology, vol. 55, no. 2, pp. 238-244, 2006.

[15] L. Manolache and V. Benea, "Stress in patients with alopecia areata and vitiligo," Journal of the European Academy of Dermatology and Venereology, vol. 21, no. 7, pp. 921-928, 2007.

[16] L. Manolache, D. Petrescu-Seceleanu, and V. Benea, "Correlation of stressful events with onset of vitiligo in children," Journal of the European Academy of Dermatology and Venereology, vol. 23, no. 2, pp. 187-188, 2009.

[17] S. Uncu, S. Yayli, S. Bahadir, A. Ökiten, and K. Alpay, "Relevance of autoimmune thyroiditis in children and adolescents with vitiligo," International Journal of Dermatology, vol. 50, no. 2, pp. 175-179, 2011.

[18] C. Vrijman, M. W. Kroon, J. Limpens et al., "The prevalence of thyroid disease in patients with vitiligo: a systematic review," British Journal of Dermatology, vol. 167, no. 6, pp. 1224-1235, 2012.

[19] D. L. Jacobson, S. J. Gange, N. R. Rose, and N. M. H. Graham, "Epidemiology and estimated population burden of selected autoimmune diseases in the United States," Clinical Immunology and Immunopathology, vol. 84, no. 3, pp. 223-243, 1997.

[20] D. S. Dean and H. Gharib, "Epidemiology of thyroid nodules," Best Practice and Research: Clinical Endocrinology and Metabolism, vol. 22, no. 6, pp. 901-911, 2008.

[21] R. Rostami, M. R. Aghasi, A. Mohammadi, and J. NouroozZadeh, "Enhanced oxidative stress in Hashimoto's thyroiditis: inter-relationships to biomarkers of thyroid function," Clinical Biochemistry, vol. 46, no. 4-5, pp. 308-312, 2013.

[22] E. Ademoğlu, N. Özbey, Y. Erbil et al., "Determination of oxidative stress in thyroid tissue and plasma of patients with Graves' disease," European Journal of Internal Medicine, vol. 17, no. 8, pp. 545-550, 2006.

[23] A. A. Shah and A. A. Sinha, "Oxidative stress and autoimmune skin disease," European Journal of Dermatology, vol. 23, no. 1, pp. 5-13, 2013.

[24] L. F. Eichenfield, W. L. Tom, S. L. Chamlin et al., "Guidelines of care for the management of atopic dermatitis: section 1 . Diagnosis and assessment of atopic dermatitis Work Group," Journal of the American Academy of Dermatology, vol. 70, no. 2, pp. 338-351, 2014. 


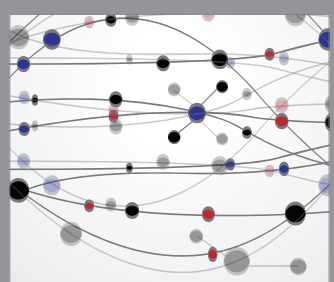

The Scientific World Journal
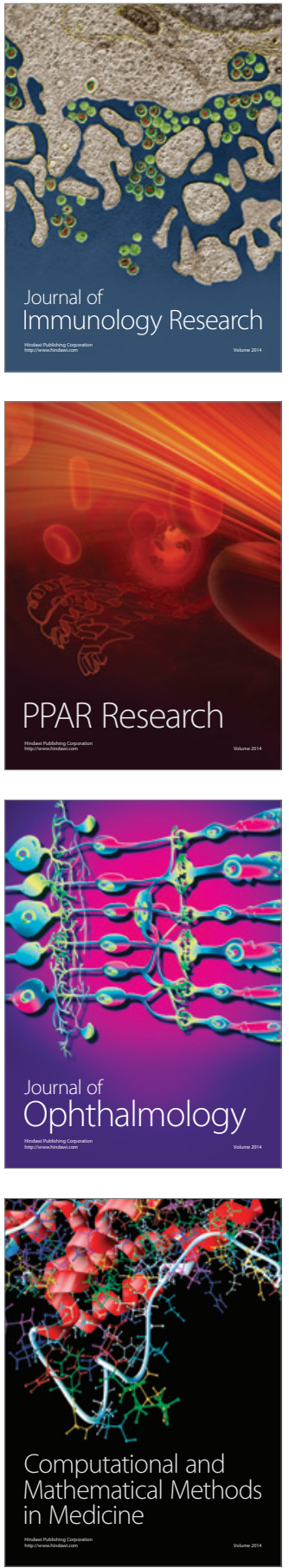

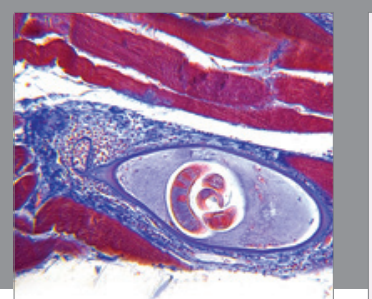

Gastroenterology Research and Practice

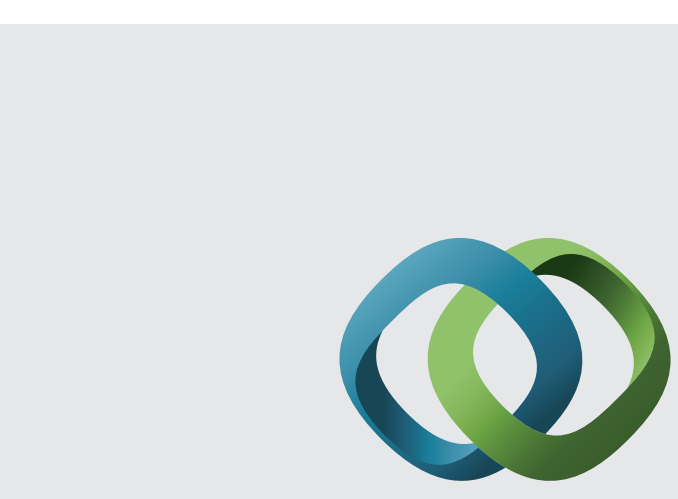

\section{Hindawi}

Submit your manuscripts at

http://www.hindawi.com
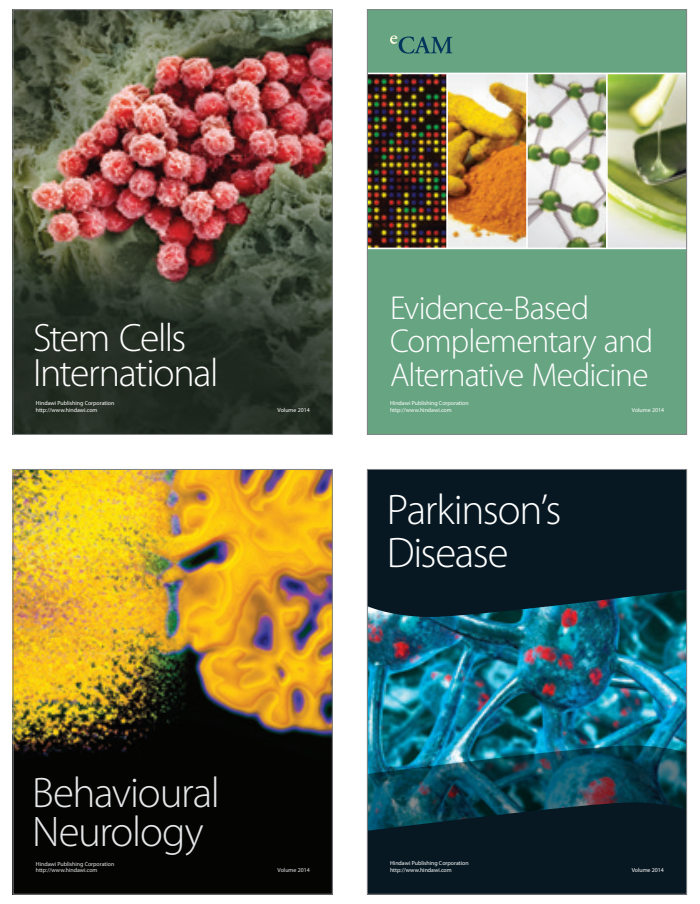
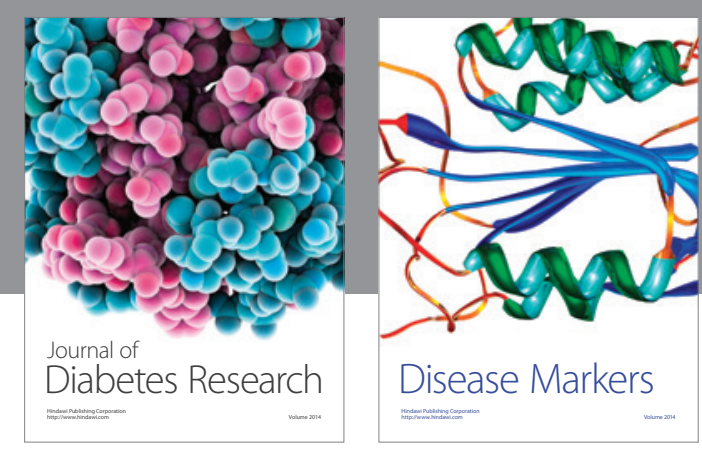

Disease Markers
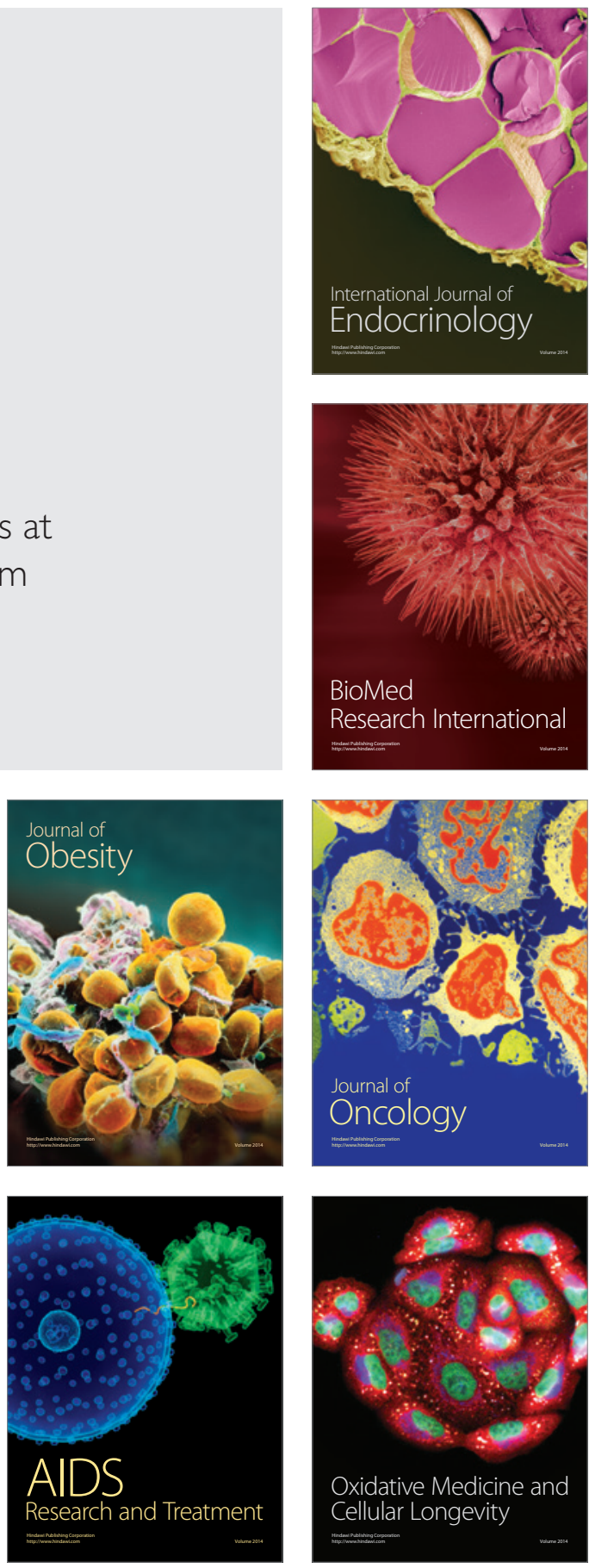